\title{
Selection of Suspicious ROIs in Breast DCE-MRI
}

\author{
Roberta Fusco ${ }^{1,3}$, Mario Sansone ${ }^{1}$, Carlo Sansone ${ }^{2}$, and Antonella Petrillo ${ }^{3}$ \\ 1 Dipartimento di Ingegneria Biomedica, Elettronica e delle Telecomunicazioni \\ Universitá 'Federico II' di Napoli, Italia \\ 2 Dipartimento di Informatica e Sistemistica \\ Universitá 'Federico II' di Napoli, Italia \\ 3 Dipartimento di Diagnostica per Immagini
}

Istituto Nazionale dei Tumori 'Fondazione Pascale' Napoli, Italia

\begin{abstract}
Dynamic Contrast Enhanced Magnetic Resonance Imaging (DCE-MRI) could be helpful in screening high-risk women and in staging newly diagnosed breast cancer patients. Selection of suspicious regions of interest (ROIs) is a critical pre-processing step in DCE-MRI data evaluation. The aim of this work is to develop and evaluate a method for automatic selection of suspicious ROIs for breast DCE-MRI. The proposed algorithm includes three steps: (i) breast mask segmentation via intensity threshold estimation; (ii) morphological operations for hole-filling and leakage removal; (iii) suspicious ROIs extraction. The proposed approach has been evaluated, using adequate metrics, with respect to manual ROI selection performed, on ten patients, by an expert radiologist.
\end{abstract}

Keywords: DCE-MRI, Breast, ROI selection, segmentation.

\section{Introduction}

Breast cancer is the most common cancer type among women in the Western world. It is the second leading cause of cancer death in women today (after lung cancer) and is estimated to cause $15 \%$ of cancer deaths [1].

The currently widespread screening method is RX mammography [2]; however, Dynamic Contrast Enhanced Magnetic Resonance Imaging (DCE-MRI) has demonstrated a potential in screening of high-risk women, in staging newly diagnosed breast cancer patients and in assessing therapy effects [1] thanks to its minimal invasiveness and to the possibility to visualize functional information not available with conventional imaging.

Breast DCE-MRI examination involves the imaging of the breast before and after the injection of a contrast agent (a commonly used tracer is Gd-DTPA, administered intravenously). DCE-MRI data consist in one pre-contrast series of T1-weighted images (images with greater signal intensity from fat-containing tissues and where most of the contrast between tissues is due to differences in the Spin-lattice relaxation time known as T1 value 3] spanning both breasts, followed by a fixed number of post-contrast series. 
Highly vascularised regions, such as tumors, exhibit typical patterns of signal enhancement vs. time as described in [4: typically, a quick tracer uptake (III,IV,V), is followed by a plateau (IV) or washout (V) (Fig 1); normal or benign tissues are characterised by either no enhancement (I) (especially in predominantly adipose regions) or slower enhancement with delayed washout (II).

By analyzing signal intensity-time curves, it is possible to characterize each voxel and detect abnormalities within the breast [5].

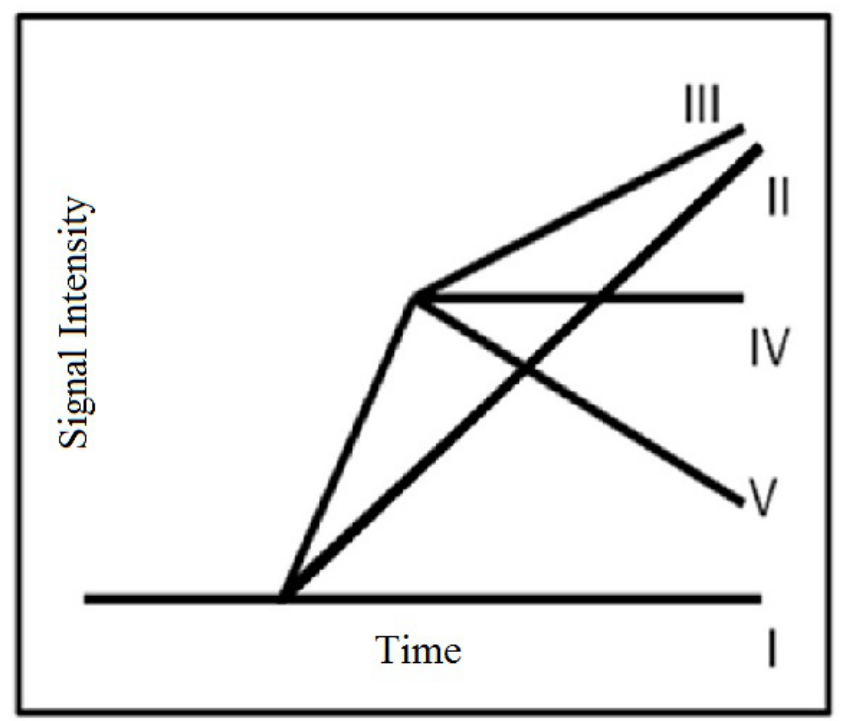

Fig. 1. Types of dynamic signal enhancement curves

Manual selection of suspicious regions of interest (ROIs), which is the critical first step in lesion detection and evaluation, is operator-dependent and time consuming. Moreover, given the vast quantity and multidimensionality of data to be analysed in a DCE MRI data set, the possibility exists that diagnostically significant regions of enhancement may be overlooked 667 .

Therefore, lesion detection for breast DCE-MRI is a difficult task that can be supported by automatic procedures for identification of suspicious ROIs.

For example, while the main objective of Tzacheva et al. 8] was classification of malignant breast lesions, they used a very simple ROI selection procedure based only on high signal intensity but they did not specify how the corresponding threshold has been chosen; moreover in their study only static features were used without taking advantage of the whole dynamic information.

As another example, Lucht et al. [9] used pharmacokinetic modelling to produce parametric maps which were used for manual ROI selection; however, the computation of tracer kinetic parameters is still a lengthy task when performed on entire images. 
Gal et al. 7] proposed a region growing approach based both on original image intensity values and fitted pharmacokinetic parameters. This approach involves the automatic identification of a seed voxel. Their results indicate a high sensitivity. However, ROI selection using kinetic parameters only (e.g. via thresholding) is an improper approach because tumor heterogeneity, which has been shown to be an important factor to be accounted for, could be missed.

From previous considerations, it emerges that an optimal automatic algorithm for selection of suspicious ROIs should have the following characteristics: it should take advantage of the whole dynamic information (not only static images), it should not miss tumor heterogenity and it should be fast enough to be applied as a first step to more sophisticated classification procedures.

The aim of this work is to propose an automatic method for suspicious ROI selection within the breast using dynamic-derived information from DCE-MRI data.

Our approach is different from the previous ones because it does not involve the lengthy computation of pharmacokinetic parameters but at the same time it exploits the whole dynamic information contained in the time-intensity curves by means of simple dynamic-derived characteristics.

In this study we evaluated the performances of the proposed method using the results of manual segmentation (gold standard) performed by an expert radiologists on ten histologically proven breast lesions ( 5 benign and 5 malignant). The results of the proposed method were compared to the gold standard using opportune metrics of segmentation accuracy.

The paper is organized as follows. In Section 2 we describe the characteristics of recruited patients, the breast DCE-MRI data acquisition protocol and the proposed automatic suspicious ROI selection algorithm. The obtained results are presented in Section 3 and discussed in Section 4, where we also draw some conclusions.

\section{Materials and Methods}

\subsection{Patient Selection}

Ten women (average age 40 years) with benign or malignant lesions histopathologically proven were enrolled (Table 1). Five cases were malignant (2 ductal carcinoma in situ, DCIS; 2 invasive ductal carcinoma, IDC; 1 invasive lobular carcinoma, ILC) and five cases were benign (4 fibroadenomata, 1 atypical ductal hyperplasia).

\subsection{Data Acquisition}

The patients underwent imaging with a $1.5 \mathrm{~T}$ scanner (Magnetom Symphony, Siemens Medical System, Erlangen, Germany) equipped with a phased-array body coil. DCE T1-weighted FLASH 3-D coronal images were acquired (TR/TE: 9.8/4.76 ms; flip angle: 25 degrees; field of view 330x247 mmxmm; matrix: 
Table 1. Patients characteristics

\begin{tabular}{|c|c|l|l|}
\hline Patient ID & Age & Pathology & Cancer Type \\
\hline 1 & 37 & Malignant & IDC \\
2 & 39 & Benign & Fibroadenomata \\
3 & 47 & Malignant & ILC \\
4 & 67 & Malignant & DCIS \\
5 & 27 & Malignant & DCIS \\
6 & 37 & Benign & Fibroadenomata \\
7 & 41 & Benign & Fibroadenomata \\
8 & 36 & Benign & ADH \\
9 & 33 & Benign & Fibroadenomata \\
10 & 40 & Malignant & IDC \\
\hline
\end{tabular}

256x128; thickness: 2 mm; gap: 0; acquisition time: $56 \mathrm{~s}$; 80 slices spanning entire breast volume). One series was acquired before and 9 series after intra-venous injection of $2 \mathrm{ml} / \mathrm{kg}$ body weight of a positive paramagnetic contrast medium (Gd-DOTA, Dotarem, Guerbet, Roissy CdG Cedex, France). Automatic injection system was used (Spectris Solaris EP MR, MEDRAD, Inc.,Indianola, PA) and injection flow rate was set to $2 \mathrm{ml} / \mathrm{s}$ followed by a flush of $10 \mathrm{ml}$ saline solution at the same rate.

\subsection{Manual Segmentation}

The manual segmentation was performed by an expert radiologist on the fatsuppressed image obtained subtracting the basal pre-contrast image from the 5th post-contrast image. Per each patient all the slices including the lesion have been used. The segmentation was performed by means of the OsiriX v.3.8.1 3 software 3 .

\subsection{ROI Selection}

The proposed algorithm includes three steps.

The first step involves Breast Mask (BM) extraction by means of automatic intensity threshold estimation (Otsu Thresholding) [10] on the parametric map obtained considering the sum of intensity differences (SOD) calculated pixel by pixel. In fact, this parameter describes the dynamic information of the whole curve and reflects the history of contrast agent enhancement with time [1]:

$$
S O D_{p}=\operatorname{Pre}_{p}+\sum_{i=1}^{T}\left|\operatorname{Post}_{p}(i)-\operatorname{Post}_{p}(i-1)\right|
$$

where $S O D_{p}$ is the SOD for the $p$ pixel; $\operatorname{Pre}_{p}$ is the pre-contrast intensity; $\operatorname{Post}_{p}(i)$ is $i$-th post-contrast scan and $T$ is the total number of scans.

The second step includes hole-filling and leakage removal by means of morphological operators: 'closing' is required to fill the holes on the boundaries of 
breast mask; 'filling' is required to fill the holes within the breasts; 'erosion' is required to reduce the dilation obtained by the closing operation [12].

The third step includes suspicious ROIs extraction. The dynamical features of each pixel are analysed. A pixel is assigned to suspicious ROI if it satisfies two conditions: the maximum of its normalized time-intensity curve (as Figure 2 shows) should be grater than 0.3 and the maximum signal intensity should be reached before the end of the scan time. The first condition assures that the pixels within the ROI have a significant contrast agent uptake (thus excluding type I and type II curves) and the second condition is required for the time-intensity pattern to be of type IV or V (thus excluding type III curves) [13 14 15].

The choice of the threshold 0.3 was based on the findings by [16] : in fact, in their study lesions with TIC enhancement less than $50 \%$ above the baseline were considered non-tumoral; they also noticed that lowering the threshold to $40 \%$ improved the accuracy of diagnosis. We proposed a threshold of $30 \%$ in order to reduce the number of false negatives.

All procedures were implemented in Matlab R2008a using Image toolbox.
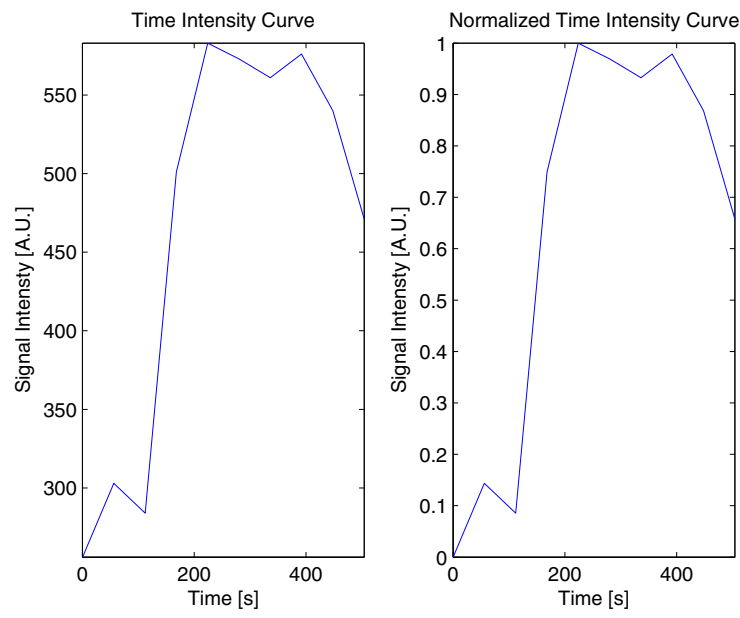

Fig. 2. Time Intensity Curve vs Normalized Time Intensity Curve: an example of a time intensity curve was reported on the left, while an example of a normalized time intensity curve with respect to the maximum signal intensity was reported on the right

\subsection{Evaluation}

According to the approach proposed by [17] the accuracy of a segmentation method can be evaluated calculating the following quantities (BM is the Breast Mask): the Overlap (O) and the Union (U) between the ground truth $S_{m}$ and the automatic segmentation $S_{c}$; the true positive fraction (TPF); the false negative fraction $(\mathrm{FNF})$; the true negative fraction $(\mathrm{TNF})$; the false positive fraction $(\mathrm{FPF})$; the accuracy (ACC): 


$$
\begin{aligned}
O & =S_{m} \bigcap S_{c} \\
U & =S_{m} \bigcup S_{c} \\
T P F & =\frac{O}{S_{m}} \\
T N F & =\frac{B M-U}{B M-S_{m}} \\
F N F & =\frac{S_{m}-S_{c}}{S_{m}}=1-T P F \\
F P F & =\frac{S_{m}-O}{B M-S_{m}}=1-T N F \\
A C C & =\frac{T P F+T N F}{T P F+T N F+F P F+F N F}
\end{aligned}
$$

It is clear that only two measures are independent and are required to quantify the accuracy of the method.

\section{Experimental Results}

Figure 3 shows the result of a manual ROI lesion selection onto a fat-suppressed image.

Figure 4 (a) shows the results of the first step, Breast mask selection after Otsu thresholding on SOD feature. Figure 4 (b) shows the breast mask after morphological operators (closing, filling and erosion). Figure 4 (c) shows the result of automatic suspicious ROI selection.

Table 2 reports the results of the evaluation study. Per each patient the TPF, TNF and ACC have been reported.

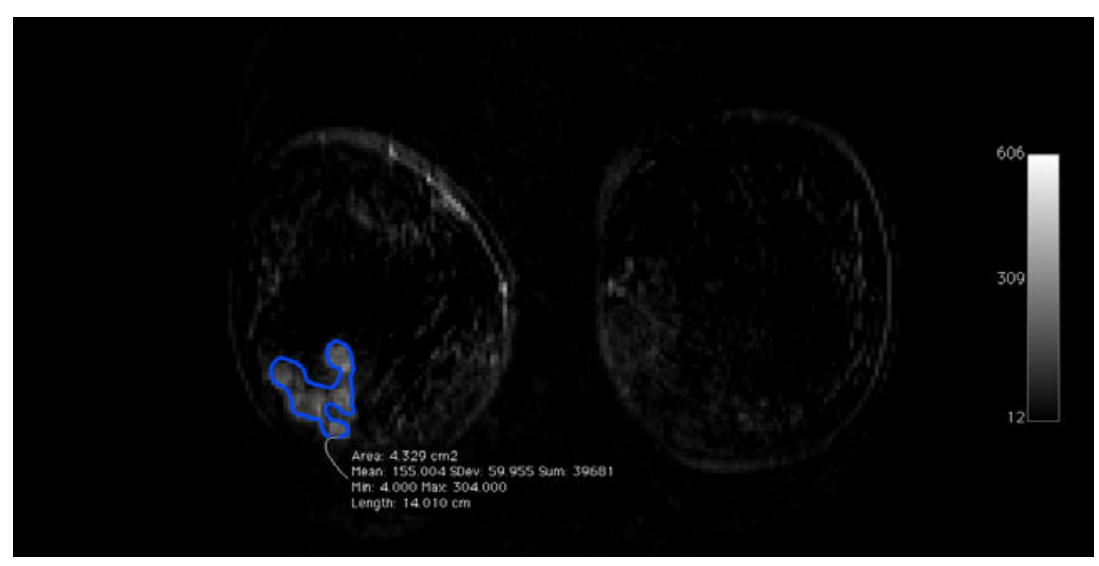

Fig. 3. Manual selection of suspicious ROI 


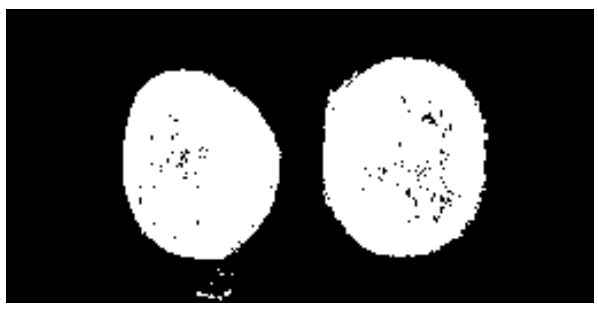

(a)

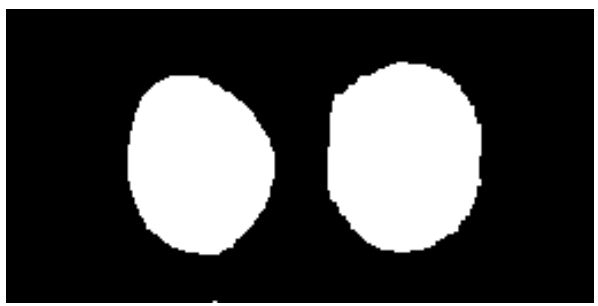

(b)

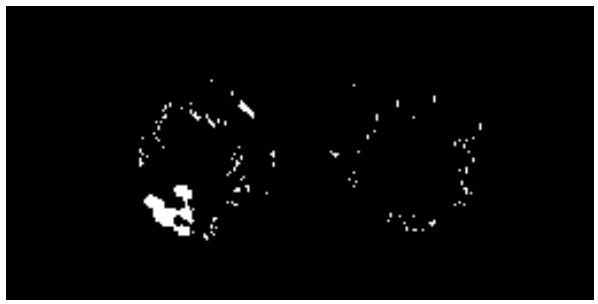

(c)

Fig. 4. Results of automatic ROI selection: (a) breast mask selection via Otsu thresholding on SOD; (b) breast mask after morphological operators (closing, filling and erosion); (c) automatic suspicious ROI selection 
Table 2. ROI selection results

\begin{tabular}{|c|r|r|r|}
\hline Patient ID & TPF & TNF & ACC [\%] \\
\hline 1 & 0.887 & 0.999 & 93.8 \\
2 & 0.799 & 0.999 & 90.0 \\
3 & 0.750 & 0.999 & 87.7 \\
4 & 0.670 & 0.999 & 83.0 \\
5 & 0.750 & 0.999 & 87.6 \\
6 & 0.580 & 0.999 & 78.8 \\
7 & 0.750 & 0.999 & 87.0 \\
8 & 0.827 & 0.999 & 90.9 \\
9 & 0.730 & 0.999 & 86.9 \\
10 & 0.879 & 0.990 & 93.0 \\
Average & 0.762 & 0.998 & 87.9 \\
\hline
\end{tabular}

\section{Discussion and Conclusions}

In this study we have proposed an algorithm for selection of suspicious ROIs on breast DCE-MRI data. The proposed algorithm is based on dynamic-derived features. The performances of the proposed method have been evaluated with respect to manual selection of an expert radiologist on a dataset of ten breast lesions.

It is worth noting that previous studies 1819 20 have also performed the breast DCE-MRI segmentation on restricted population (from thirteen to four patients) because of the difficulty to enroll patients with the same data acquisition protocol and the absence of a public database.

Our results indicate that the TPF of our automatic selection varied in the range 0.580 to 0.887 (average 0.762 ) and then the accuracy of authomatic suspicious ROI selection increases when invasive lobular o ductal cancer are considered. In fact, in those cases the lesion is larger with a greater contrast agent uptake: this determines a lower number of misclassified pixels (suspicious or not suspicious). On the contrary, the accuracy decreases when ductal carcinoma in situ (because of their size) and benign lesion are considered (because of their lower contrast agent uptake).

Selection of suspicious ROIs is to be considered as a preliminary step in tumour evaluation before more sophisticated algorithms for tissue malignancy classification. Our preliminary results show that selection of suspicious ROIs in the breast is feasible using a simple and fast algorithm based on the whole dynamic information contained in DCE-MRI. While the fraction of false positives is almost zero, further investigation is required in order to reduce the number of lost pixels potentially suspicious: this could be accomplished, for example, introducing the analysis of non-dynamic images. In the future, our preliminary study will be extended on a larger number of patients and manual segmentation will be done by multiple readers. 


\section{References}

1. Lehman, C.D., Gatsonis, C., Kuhl, C.K., Hendrick, R.E., Pisano, E.D., Hanna, L., Peacock, S., Smazal, S.F., Maki, D.D., Julian, T.B., DePeri, E.R., Bluemke, D.A., Schnall, M.D.: MRI evaluation of the contralateral breast in women with recently diagnosed breast cancer. The New England Journal of Medicine 356, 1295-1303 (2007)

2. Olsen, O., Gøtzsche, P.C.: Screening for breast cancer with mammography, Cochrane Database of Systematic Reviews, p. CD001877 (2001) (Online)

3. McRobbie, D., et al.: MRI, From picture to proton (2003)

4. Daniel, L., et al.: Breast Disease: Dynamic Spiral MR Imaging. Radiology 209, 499-509 (1998)

5. Eyal, E., Degani, H.: Model based and Model free parametric analysis of breast dynamic contrast enhanced MRI. NMR Biomed. (2007)

6. Liney, G.P., Sreenivas, M., Gibbs, P., Garcia-Alvarez, R., Turnbull, L.W.: Breast lesion analysis of shape technique: semiautomated vs. manual morphological description. Journal of Magnetic Resonance Imaging: JMRI 23, 493-498 (2006)

7. Gal, Y., Mehnert, A., Bradley, A., McMahon, K., Crozier, S.: Automatic Segmentation of Enhancing Breast Tissue in Dynamic Contrast-Enhanced MR Images. In: 9th Biennial Conference of the Australian Pattern Recognition Society on Digital Image Computing Techniques and Applications (DICTA 2007), Glenelg, Australia, pp. 124-129 (2007)

8. Tzacheva, A.A., Najarian, K., Brockway, J.P.: Breast cancer detection in gadolinium-enhanced MR images by static region descriptors and neural networks. J. Magn. Reson. Imaging 17(3), 337-342 (2003)

9. Lucht, R.E.A., Knopp, M.V., Brix, G.: Classification of signal time curves from dynamic MR mammography by neural networks. Magn. Reson. Imaging, 51-57 (2001)

10. Lee, S.H., Kim, J.H., Park, J.S., Chang, J.M., Park, S.J., Jung, Y.S., Moon, W.K.: Computerized Segmentation and Classification of Breast Lesions Using Perfusion Volume Fractions in Dynamic Contrast-enhanced MRI. In: International Conference on BioMedical Engineering and Informatics, pp. 58-62. IEEE Computer Society, Los Alamitos (2008)

11. Twellmann, T., Lichte, O., Nattkemper, T.W.: An adaptive tissue characterization network for model-free visualization of dynamic contrast-enhanced magnetic resonance image data. IEEE Trans. Med. Imaging 3, 21 (2005)

12. Chen, W., Giger, M.L., Li, H., Bick, U., Newstead, G.M.: Volumetric texture analysis of breast lesions on contrast-enhanced magnetic resonance images. Magn. Reson. Med. (2007)

13. Issa, Improved discrimination of breast lesions using selective sampling of segmented MR images, MAGMA (2006)

14. Meinel, L.A., Buelow, T., Huo, D., Shimauchi, A., Kose, U., Buurman, J., Newstead, G.: Robust segmentation of mass-lesions in contrast-enhanced dynamic breast MR images. Journal of Magnetic Resonance Imaging: JMRI 32, 110-119 (2010)

15. Sinha, U., Sinha, S., Lucas-Quesada, F.A.: Segmentation strategies for breast tumors from dynamic MR images. Journal of Magnetic Resonance Imaging 6, 753-763

16. Torricelli, P., Pecchi, A., Luppi, G., Romagnoli, R.: Gadolinium-enhanced MRI with dynamic evaluation in diagnosing the local recurrence of rectal cancer. Abdominal Imaging 28, 19-27 (2003) 
17. Udupa, J.K., Leblanc, V.R., Zhuge, Y., Imielinska, C., Schmidt, H., Currie, L.M., Hirsch, B.E., Woodburn, J.: A framework for evaluating image segmentation algorithms. Computerized Medical Imaging and Graphics: The Official Journal of the Computerized Medical Imaging Society 30, 75-87 (2006)

18. Woods, B.J., Clymer, B.D., Kurc, T., Heverhagen, J.T., Stevens, R., Orsdemir, A., Bulan, O., Knopp, M.V.: Malignant-lesion segmentation using 4D co-occurrence texture analysis applied to dynamic contrast-enhanced magnetic resonance breast image data. J. Magn. Reson. Imaging 25(3), 495-501 (2007)

19. Hill, A., Mehnert, A., Crozier, S.: Edge intensity normalization as a bias field correction during balloon snake segmentation of breast MRI. In: Conf. Proc. IEEE Eng Med Biol Soc., pp. 3040-3043 (2008)

20. Cui, Y., Tan, Y., Liberman, L., Parbhu, R., Kaplan, J., Schwartz Malignant, L.H.: lesion segmentation in contrast-enhanced breast MR images based on the markercontrolled watershed. Med. Phys. 36, 4359 (2009) 